\title{
A rare case report: successful outcome of a patient at near term pregnancy with maternal chronic obstructive hydrocephalus due to tubercular meningitis
}

\author{
Sowmya Mahesh*, Himshweta Srivastava
}

Department of Obstetrics and Gynaecology, UCMS and GTB Hospital, New Delhi, India

Received: 02 September 2019

Accepted: 01 October 2019

\section{*Correspondence:}

Dr. Sowmya Mahesh,

E-mail: sowmya_m26@yahoo.com

Copyright: () the author(s), publisher and licensee Medip Academy. This is an open-access article distributed under the terms of the Creative Commons Attribution Non-Commercial License, which permits unrestricted non-commercial use, distribution, and reproduction in any medium, provided the original work is properly cited.

\begin{abstract}
The incidence of tubercular meningitis leading to obstructive hydrocephalus is extremely rare accounting for $1 \%$ cases and is even rarer during pregnancy. A 20 -year-old $2^{\text {nd }}$ Gravida presented at 32 weeks gestation with complaints of intermittent headache, decreased vision since 3 months and irrelevant talks and agitated behavior since 1 day. On examination patient had stable vital signs but constant irritable behavior. Obstetric examination revealed a pregnancy corresponding to 32 weeks with a live fetus. Patient had a past history of Anti-tubercular therapy taken 2 years back for tubercular meningitis. Neurological examination revealed a GCS of 12/15 and Ophthalmologic examination revealed patient to be having only Perception of Light with optic atrophy on fundoscopy. NCCT head revealed moderate dilatation of bilateral ventricles and 3rd ventricle without any periventricular ooze suggestive of Chronic Obstructive Hydrocephalus. Supportive therapy was instituted with Dexamethasone and Mannitol infusion after Neurosurgical consultation along with antenatal care. Decision for termination of pregnancy was taken at 35 weeks by an elective caesarean section with simultaneous ventriculo-peritoneal shunt insertion by neurosurgical team under general anesthesia. A live male baby of $2.25 \mathrm{~kg}$ was delivered. Patient with baby was discharged on $10^{\text {th }}$ postoperative day. Obstructive hydrocephalous developing post tubercular meningitis in pregnancy is a rare event. Caesarean section was done along with shunt surgery in our case. Very few cases have been reported in the medical literature so far.
\end{abstract}

Keywords: Anti-tubercular therapy, Cerebral aqueduct, Chronic obstructive hydrocephalus, Intracranial pressure, Ventriculo-peritoneal shunt, NCCT head

\section{INTRODUCTION}

Hydrocephalus is a neurosurgical disorder with major constraints both in paediatric and adult population. ${ }^{1}$ The overall prevalence of hydrocephalus is $0.9-1.2 / 1.000 .^{2}$ There are two subtypes as communicating and noncommunicating (obstructive) hydrocephalus. Cerebral (Sylvian) aqueduct is the commonest intraventricular site for obstructive hydrocephalus. ${ }^{3}$ Cerebral aqueduct stenosis contributes $6-66 \%$ of paediatric onset and 5-49\% of adult-onset hydrocephalus., ${ }^{4,5}$ Cerebral aqueduct stenosis is a dynamic event which is affected by multiple on-going intracranial changes during pregnancy when multiple physiological changes are already taking place. The excess supratentorial cerebrospinal fluid (CSF) may disrupt the already tight aqueduct. Etiology is diverse as infectious (bacterial, viral), genetic X-linked, central nervous system malformations, head traumas, tumours, and hemorrhage. ${ }^{3}$ However, only one out of each four cases have an underlying identifiable pathology for stenosis and hence the remaining cases are named as "idiopathic." 
The ventriculo-peritoneal (VP) shunt is an effective and mainstay of treatment for hydrocephalus. Although VP shunt considerably reduces the morbidity and mortality of hydrocephalus, it often malfunctions due to complications such as obstruction, breakage, migration and infection. In pregnant women, shunt malfunction occurs due to increasing abdominal pressure associated with enlargement of the uterus and other organs.

Here, we present a case in which the patient presented to obstetrics and gynaecology casualty in in the third trimester of pregnancy and was taken up for an elective $\mathrm{C}$-section and right ventriculo-peritoneal shunt insertion under general anaesthesia after an extensive work-up.

\section{CASE REPORT}

A 20-Year-old $2^{\text {nd }}$ Gravida resident of Bulandshahar, Uttar Pradesh referred from dispensary presented to Guru Teg Bahadur Hospital Casualty on $18^{\text {th }}$ July 2018 with primary complaints of occasional pain abdomen, headache and decreased vision since 3 months duration and irrelevant talks and agitated behaviour for 1 day. She had no complaint of nausea or gait disturbances. She was improperly investigated in her antenatal period in the present pregnancy. The patient was a second gravida with a previous normal vaginal delivery 4-year years back. No history of abortions spontaneous or induced. Her past history revealed that patient had a history of tubercular meningitis 2 years back for which she had previously taken anti-tubercular therapy. Patient was restarted on ATT 1 month back in view of resurgence of symptoms of headache and patient was in Initiation Phase of ATT when she presented to us. She had no history of trauma. She had undergone an MRI in April 2018 which revealed a focal lesion of $2.4 \times 2.5 \times 2.5 \mathrm{~cm}$ in the superior cerebellar vermis and tectal region causing obstruction in the fourth ventricle causing and obstructive non-communicating moderate hydrocephalus (? infective pathology) for which the patient did not take any medicine, neurology or neurosurgical opinion.

On examination, she was conscious, confused with inadvertent talking and irritable behaviour GCS12/15(E4V4M4). Her vitals were stable. Her blood pressure was $122 / 80 \mathrm{mmHg}$ and pulse rate of $88 \mathrm{bpm}$. She presented with a gestational age of 32 weeks 5 days which was calculated from a 9-week scan as her exact LMP was not known to her. Her per abdominal examination corresponded to a gestational of age 32 weeks. It was soft and relaxed on palpation. Her FHR was $136 \mathrm{bpm}$ regular in rhythm. Per vaginal examination demonstrated a multiparous os. No evidence of any bleeding or leaking. She was admitted in our septic labour room routine investigations, basic blood work up and an obstetric scan were done.

Basic blood work up revealed haemoglobin of 10.3 $\mathrm{gm} / \mathrm{dL}$. Her liver function tests, renal function tests and serum electrolytes were within normal range. Her coagulation profile was normal. Her viral markers were negative. Her obstetric scan revealed a live fetus with parameters corresponding to 32 weeks of gestation. Liquor amnii was adequate and placenta was fundo posteriorly located. No evidence of any retroplacental clot.

\section{NCCT head images of the patient}

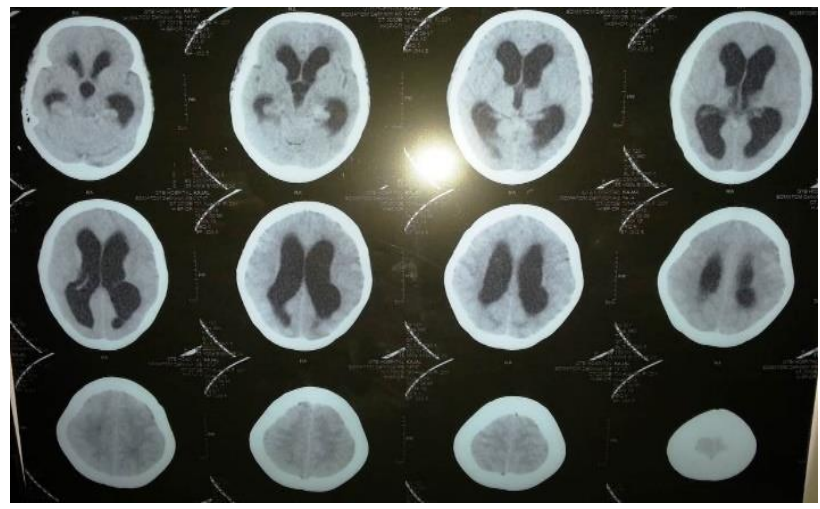

Figure 1: CT scan showing obstructive hydrocephalus.

Description: The above image shows moderate dilatation of bilateral ventricles and 3rd ventricle without any periventricular ooze suggestive of chronic obstructive hydrocephalus.

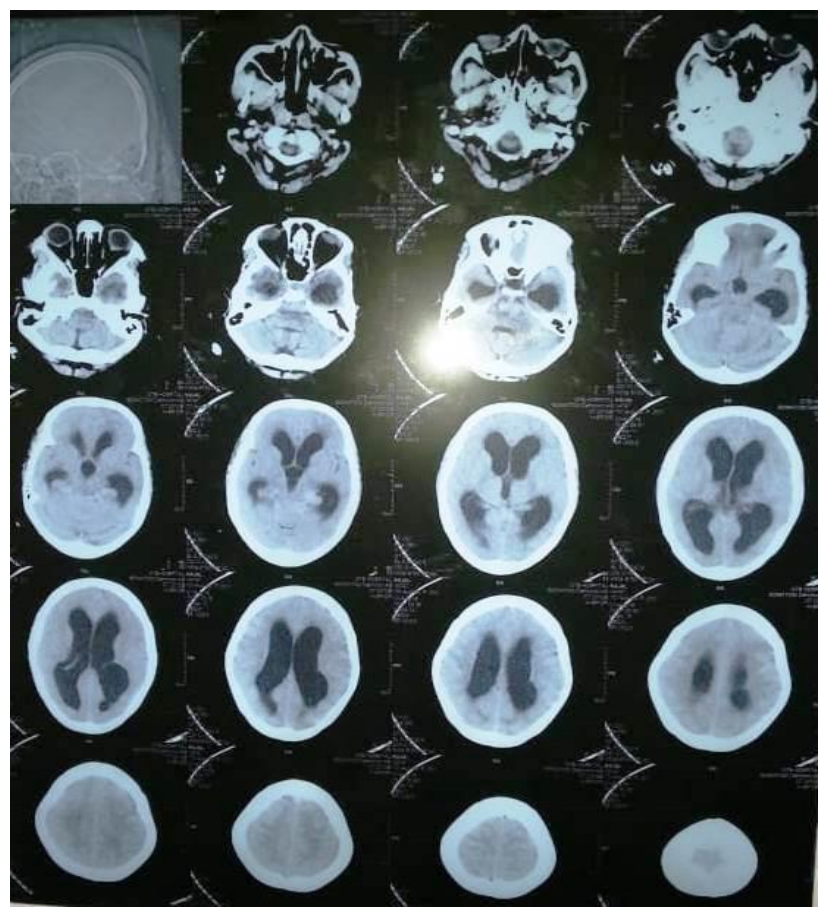

Figure 2: CT scan showing obstructive hydrocephalus.

Description: The above CT scan image shows the dilatation of frontal, temporal and occipital horns of lateral ventricles). 
An NCCT head was done in our hospital on $1^{\text {st }}$ August 2018 which revealed moderate dilatation of bilateral ventricles and $3^{\text {rd }}$ ventricle without any periventricular ooze suggestive of chronic obstructive hydrocephalus.

Ophthalmological examination revealed was only PL+ and local examination revealed bilateral lateral rectus palsy and fundus examination revealed bilateral optic nerve atrophy. Medicine referral advised dexamethasone coverage. A DOTS opinion advised us to get CBNAAT analysis of the CSF sample. Neurology referral advised us to get CSF for proteins, sugar cytology, ZN and Gram staining along with CBNAAT Analysis and advised a neurosurgery opinion. Neurosurgical opinion advised us to continue Dexamethasone $4 \mathrm{mg}$ TDS as before, IV mannitol was added and patient was to be taken up for VP shunt surgery as an elective procedure.

Patient was put on Dexamethasone coverage. Preanaesthetic work up was done and patient was to be taken up for elective LSCS with PPIUCD insertion at 36 weeks and simultaneous insertion of a VP shunt on the same sitting. On $3^{\text {rd }}$ August 2018, patient was posted for elective LSCS and Right VP shunt insertion in view of resurgent tubercular meningitis causing noncommunicating obstructive hydrocephalus. Patient delivered an LBW male baby by elective LSCS. A curvilinear incision was given on right side of scalp and a burr hole was created. A simultaneous abdominal incision was given and tunnel passed from abdominal end to cranial end. Ventricular end connected to abdominal end and free flow of CSF noted. Incision was closed in layers. CSF sampling was done and reports revealed lymphocytosis and $\mathrm{ZN}$ staining was positive for acid fast bacilli and CBNAAT was positive.

Following VP shunt insertion, patient was shifted to Neurosurgery ICU for monitoring. After 6 days of monitoring patient was shifted back to Post-Natal Ward. We continued our regular post-operative care and patient was discharged after 10 days on $13^{\text {th }}$ August 2018. A post-delivery NCCT head was done. GCS was stable in the post-natal period. Patient was advised to continue Category 2 ATT. She was given post-natal advice regarding wound care, breast care, contraception and immunization of baby. She was also advised to follow up in the Neurosurgery and TB clinic. Patient did not report for follow up to obstetrics-gynaecology and neurosurgery OPD.

\section{DISCUSSION}

Cerebral (Sylvian) aqueduct is the most common intraventricular site for obstructive hydrocephalus. Cerebral aqueduct stenosis is a dynamic event affected with on-going intracranial changes such as pregnancy in which many physiological changes occur, especially supratentorial cerebrospinal fluid (CSF) excess may disrupt the already tight aqueduct. ${ }^{6}$ Based on this assumption, we should further understand physiological changes that occur throughout pregnancy.

Increased ICP hence facilitate obstructive hydrocephalus by further narrowing the cerebral aqueduct, which is already tight just before the pregnancy. While the brain decreases in volume (4.1-6.6\%), ventricular size increases $(17.3-29.5 \%)$ until term. From delivery to $24^{\text {th }}$ week postpartum, normalization in brain volume and ventricular size continues.

In most studies, presenting symptoms were headache (most common), nausea and/or vomiting, depressed sensorium, seizure, memory disturbance, and vision problem. ${ }^{7-9}$ Clinical presentation can be easily confused with preeclampsia. In rare cases with concomitant brain tumor, tumor-related signs and symptoms have also been observed. Therefore, imaging test in suspected cases should be conducted. In emergency circumstances, brain CT imaging is the best modality of choice. However, pregnancy is a contradiction for ionizing radiation; hence, MR imaging and neurological examination become vital tools of evaluation. Shunt systems (ventriculo-peritoneal, ventriculo-atrial and ventriculo-pleural), endoscopic third ventriculostomy and transient ventriculostomy have been preferred management tools for hydrocephalus in most patients.

After treatment of hydrocephalus, the next question to be answered is which way of delivery is the best option for the patient. If the patient is in well status without any symptoms, spontaneous vaginal delivery will be a good option. In spontaneous vaginal delivery, the second stage of labour, which needs intense straining resulting in increased ICP should be kept at minimum. In unstable patients and in cases with obstetric indications, C-Section under general anaesthesia should be conveyed with motorization of patients' fluid status, central venous pressure. In severe cases, diuretics and steroid can be used. In circumstances with controlled ICP, epidural anaesthesia can be conveyed during $\mathrm{C}$-section.

Postpartum shunt dysfunction and shunt infection risks were reported to be present until 6 months to 1 year. ${ }^{7,10}$ Hence, continuous clinical follow-ups even after the delivery are necessary to prevent such complications in a timely manner.

\section{CONCLUSION}

Obstructive hydrocephalus is a very rare complication during pregnancy. Hydrocephalus becomes obvious and necessitates treatment before the third trimester of pregnancy. Timely diagnosis, especially differentiation from preeclampsia, is a life-saving step. If no complication happens during intervention for hydrocephalus, spontaneous vaginal delivery is a safe way of delivery for both mother's and new-born's wellbeing. C-section should be saved for obstetrical indications and can be conducted under epidural 
anaesthesia if ICP is kept under control. Interdisciplinary approach of neurosurgeons and anaesthesiologists is pivotal for delicate care of the patient and the baby.

Funding: No funding sources

Conflict of interest: None declared

Ethical approval: Not required

\section{REFERENCES}

1. Stagno V, Navarrete EA, Mirone G, Esposito F. Management of hydrocephalus around the world. World Neurosurg. 2013;79(2Suppl):S23.e17-20.

2. Oi S. Classification and definition of hydrocephalusorigin, controversy and assignment of the terminology. In: Cinalli G, Sainte-Rose C, Maixner W, editors. Pediatric Hydrocephalus. Italia: SpringerVerlag; 2005:95-112.

3. Spennato P, Tazi S, Bekaert O, Cinalli G, Decq P. Endoscopic third ventriculostomy for idiopathic aqueductal stenosis. World Neurosurg. 2013;79(2 Suppl):S21.e13-20.

4. Hirsch JF, Hirsch E, Sainte Rose C, Renier D, PierreKhan A. Stenosis of the aqueduct of Sylvius. Etiology and treatment. J Neurosurg Sci. 1986;30:29-39.
5. Jellinger G. Anatomopathology of non-tumoral aqueductal stenosis. J Neurosurg Sci. 1986;30:1-16.

6. Kulkarni AV, Hui S, Shams I, Donnelly R. Quality of life in obstructive hydrocephalus: endoscopic third ventriculostomy compared to cerebrospinal fluid shunt. Childs Nerv Syst. 2010;26:75-9.

7. Monfared AH, Koh KS, Apuzzo ML, Collea JV. Obstetric management of pregnant women with extracranial shunts. Can Med Assoc J. 1979;120:562-3.

8. Howard TE, Herrick CN. Pregnancy in patients with ventriculoperitoneal shunts: Report of two cases. Am J Obstet Gynecol. 1981;141:99-101.

9. Nugent P, Hoshek S. Large extra-abdominal cyst as a postpartum complication of peritoneal shunt. Case Report J Neurosurg. 1986;64:151-2.

10. Fox BC. Delayed-onset postpartum meningitis due to group B Streptococcus. Clin Infect Dis. 1994;19:350.

Cite this article as: Mahesh $\mathrm{S}$, Srivastava $\mathrm{H}$. A rare case report: successful outcome of a patient at near term pregnancy with maternal chronic obstructive hydrocephalus due to tubercular meningitis. Int J Reprod Contracept Obstet Gynecol 2019;8:4626-9. 